(C) [2007] IEEE. Reprinted, with permission, from [Yong Zhang, Shao, K.R.; Youguang Guo; Jianguo Zhu; Xie, D.X.; Lavers,J.D., A Comparison of Point Interpolative Boundary Meshless Method Based on PBF and RBF for Transient Eddy-Current Analysis, Magnetics, IEEE Transactions on (Volume:43, Issue: 4 ), April 2007]. This material is posted here with permission of the IEEE. Such permission of the IEEE does not in any way imply IEEE endorsement of any of the University of Technology, Sydney's products or services. Internal or personal use of this material is permitted. However, permission to reprint/republish this material for advertising or promotional purposes or for creating new collective works for resale or redistribution must be obtained from the IEEE by writing to pubspermissions@ieee.org. By choosing to view this document, you agree to all provisions of the copyright laws protecting it 


\section{A Comparison of Point Interpolative Boundary Meshless Method Based on PBF and RBF for Transient Eddy Current Analysis}

\section{Yong Zhang, K.R. Shao}

Huazhong Univ. of Sci. \& Tech., Wuhan, 430074, P.R. China

\section{Youguang Guo}

University of Technology, Sydney, NSW 2007, Australia

\section{D.X.Xie}

Shenyang University of Technology, Shenyang 110023 P.R.China

$$
\text { J.D. Lavers }
$$




\section{University of Toronto, Toronto, Ont. M5S 3G4, Canada}

Abstract -- This paper presents boundary polynomial point interpolation meshless method (BPPIM) and boundary radial point interpolation meshless method (BRPIM) based on polynomial basis function (PBF) and radial basis function (RBF) respectively for transient eddy current analysis, and their interpolation shape functions satisfy the Kronecker delta function, thus the essential boundary conditions can be directly imposed on the boundary nodes. An example on analyzing transient eddy current of a square metal column is set to prove the validity of the proposed methods, and a comparison on accuracy between BPPIM and BRPIM is analyzed as well.

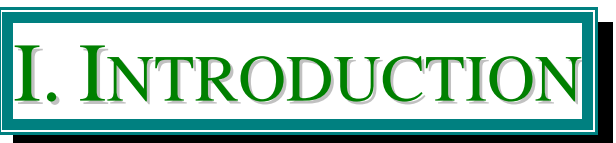


Boundary meshless methods (BMMs) are attractive and important computational techniques for reducing the dimensionality of the solving problems. Several boundary-type meshless methods have been developed for many potential and elastic problems [1] [2], they need no discretization of the boundary and are proven as robust numerical methods. But very few of them are used to solve electromagnetic problems, say nothing of transient eddy current problems. A work of BMMs for transient eddy current problems has been recently published by the authors [3]. In this paper, boundary polynomial point interpolation meshless method (BPPIM) and boundary radial point interpolation meshless method (BRPIM) based on polynomial basis function (PBF) and radial basis function (RBF) are presented respectively for transient eddy current analysis, and both their interpolation shape functions satisfy the Kronecker delta function, thus the essential boundary conditions can be 
$\mathrm{u}$ and $\mathrm{q}$ are constructed independently using PIM shape function as

$$
u(s)=\sum_{j=1}^{m} p_{j}(s) a_{j}=\mathbf{p}^{\mathrm{T}}(s) \cdot \mathbf{a} \quad q(s)=\sum_{j=1}^{m} p_{j}(s) b_{j}=\mathbf{p}^{\mathrm{T}}(s) \cdot \mathbf{b}
$$

In matrix form, there are

$$
\mathbf{a}=\left[a_{1}, a_{2}, \cdots, a_{m}\right]^{\mathrm{T}} \mathbf{b}=\left[b_{1}, b_{2}, \cdots, b_{m}\right]^{\mathrm{T}} \mathbf{p}(s)=\left[1, s, \cdots, s^{m-1}\right]^{\mathrm{T}}
$$

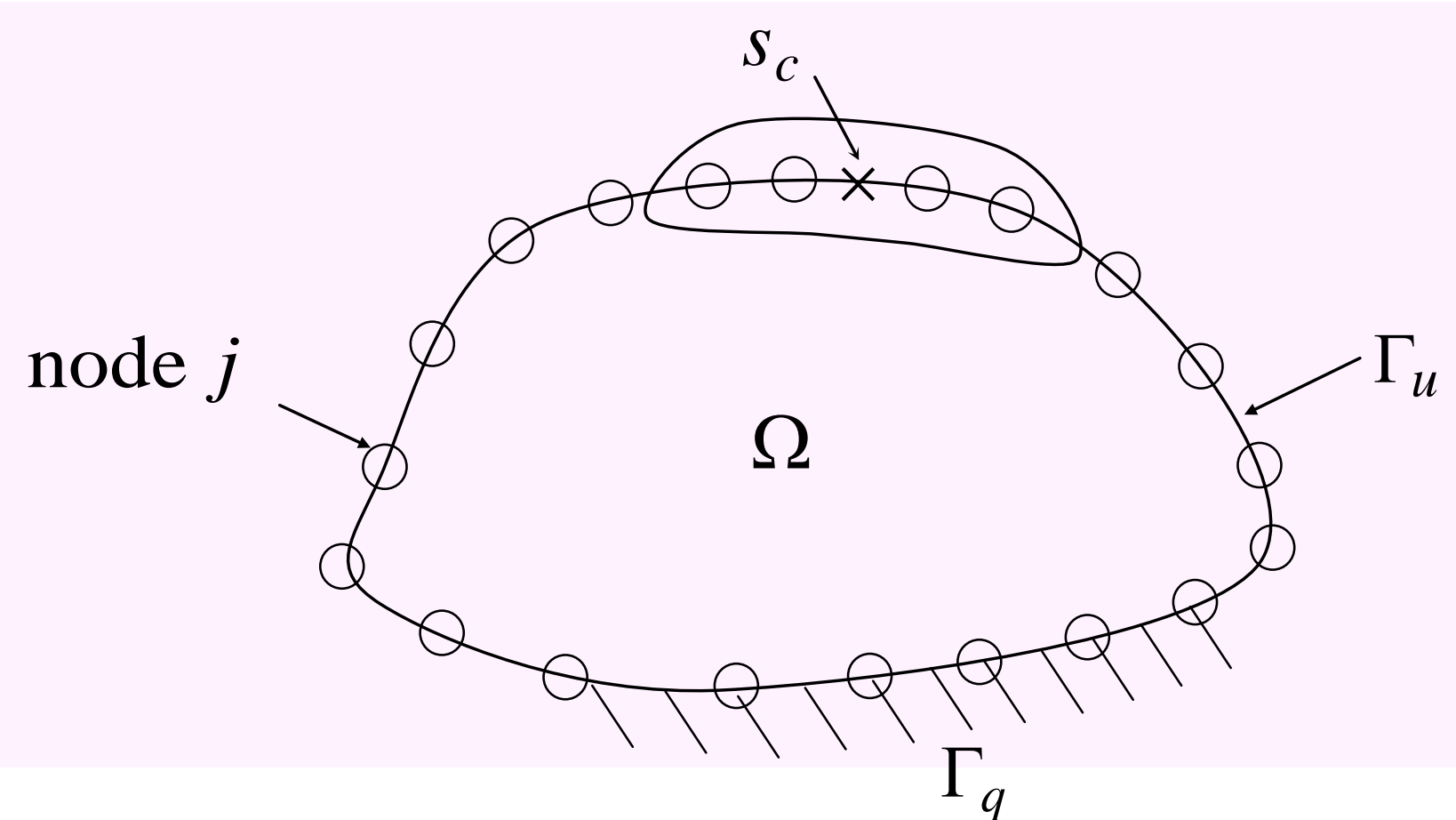

Fig.1 Problem domain and boundary and nodes 
The coefficient $a_{j}$ and $b_{j}$ in (2.a) and (2.b) can be determined by enforcing (1.a) and (1.b) to be satisfied at the $m$ nodes surrounding the point $s_{c}$. Equation (1) can then be written in the following matrix form

$$
\begin{aligned}
\mathbf{u}_{m} & =\mathbf{P}_{c} \mathbf{a} \\
\mathbf{q}_{m} & =\mathbf{P}_{c} \mathbf{b}
\end{aligned}
$$

In (3.a) and (3.b), we have

$$
\begin{aligned}
& \mathbf{u}_{m}=\left[u_{1}, u_{2}, \cdots, u_{m}\right]^{\mathrm{T}} \\
& \mathbf{q}_{m}=\left[q_{1}, q_{2}, \cdots, q_{m}\right]^{\mathrm{T}} \\
& \mathbf{P}_{c}=\left[\mathbf{p}\left(s_{1}\right), \mathbf{p}\left(s_{2}\right), \cdots, \mathbf{p}\left(s_{m}\right)\right]^{\mathrm{T}}
\end{aligned}
$$


Solving $a$ and $b$ from (3.a) and (3.b), then we can obtain

$$
\begin{aligned}
& u(s)=\boldsymbol{\Phi}^{\mathrm{T}}(s) \mathbf{u}_{m} \\
& q(s)=\boldsymbol{\Phi}^{\mathrm{T}}(s) \mathbf{q}_{m}
\end{aligned}
$$

\section{B. Radial basis point interpolation method (RPIM)}

To radial basis function point interpolation method, (1) can be rewritten as

$$
\begin{aligned}
& u(s)=\sum_{i=1}^{n} R_{i}(s) a_{i}+\sum_{j=1}^{m} p_{j}(s) b_{j}=\mathbf{R}^{\mathrm{T}}(s) \mathbf{a}+\mathbf{p}^{\mathrm{T}}(s) \mathbf{b} \\
& q(s)=\sum_{i=1}^{n} R_{i}(s) c_{i}+\sum_{j=1}^{m} p_{j}(s) d_{j}=\mathbf{R}^{\mathrm{T}}(s) \mathbf{c}+\mathbf{p}^{\mathrm{T}}(s) \mathbf{d}
\end{aligned}
$$

Where $R_{i}(s)=\left(s_{i}^{2}+c\right)^{q}$ in this paper, the parameters and basis 
in matrix form are

$$
\begin{gathered}
\mathbf{a}=\left[a_{1}, a_{2}, \cdots, a_{n}\right]^{\mathrm{T}} \mathbf{b}=\left[b_{1}, b_{2}, \cdots, b_{m}\right]^{\mathrm{T}} \mathbf{c}=\left[c_{1}, c_{2}, \cdots, c_{n}\right]^{\mathrm{T}} \\
\mathbf{R}(s)=\left[R_{1}(s), R_{2}(s), \cdots, R_{n}(s)\right]^{\mathrm{T}} \\
\mathbf{d}=\left[d_{1}, d_{2}, \cdots, d_{m}\right]^{\mathrm{T}} \quad \mathbf{p}(s)=\left[1, s, \cdots, s^{m-1}\right]^{\mathrm{T}}
\end{gathered}
$$

For the uniqueness of the radial point interpolation, the relationship should be satisfied as following

$$
\sum_{i=1}^{n} p_{j}(s) a_{i}=\mathbf{P}_{0}^{\mathrm{T}} \mathbf{a}=0 \sum_{i=1}^{n} p_{j}(s) c_{i}=\mathbf{P}_{0}^{\mathrm{T}} \mathbf{c}=0 \quad(j=1,2, \cdots, m)
$$

Combining (6) (8) get the matrix form of the parameters are

$$
\left[\begin{array}{cc}
\mathbf{R}_{\mathbf{0}} & \mathbf{P}_{\mathbf{0}} \\
\mathbf{P}_{\mathbf{0}}^{\mathbf{T}} & 0
\end{array}\right]\left[\begin{array}{l}
\mathbf{a} \\
\mathbf{b}
\end{array}\right]=\mathbf{G}\left[\begin{array}{l}
\mathbf{a} \\
\mathbf{b}
\end{array}\right]=\left[\begin{array}{l}
\mathbf{u}_{n} \\
0
\end{array}\right]\left[\begin{array}{cc}
\mathbf{R}_{\mathbf{0}} & \mathbf{P}_{\mathbf{0}} \\
\mathbf{P}_{\mathbf{0}}^{\mathbf{T}} & 0
\end{array}\right]\left[\begin{array}{l}
\mathbf{c} \\
\mathbf{d}
\end{array}\right]=\mathbf{G}\left[\begin{array}{l}
\mathbf{c} \\
\mathbf{d}
\end{array}\right]=\left[\begin{array}{l}
\mathbf{q}_{n} \\
0
\end{array}\right]
$$

And the unknown parameters are solved from (9) as 


$$
\left[\begin{array}{l}
\mathbf{a} \\
\mathbf{b}
\end{array}\right]=\mathbf{G}^{-1}\left[\begin{array}{l}
\mathbf{u}_{n} \\
0
\end{array}\right] \quad\left[\begin{array}{l}
\mathbf{c} \\
\mathbf{d}
\end{array}\right]=\mathbf{G}^{-1}\left[\begin{array}{l}
\mathbf{q}_{n} \\
0
\end{array}\right]
$$

Substituting (10) into (6) and get

$$
\begin{aligned}
& u(s)=\left[\mathbf{R}^{\mathrm{T}}(s) \mathbf{p}^{\mathrm{T}}(s)\right] \mathbf{G}^{-1}\left[\mathbf{u}_{n} 0\right]^{\mathrm{T}}=\boldsymbol{\Phi}^{\mathrm{T}}(s) \mathbf{u}_{n} \\
& q(s)=\left[\mathbf{R}^{\mathrm{T}}(s) \mathbf{p}^{\mathrm{T}}(s)\right] \mathbf{G}^{-1}\left[\mathbf{q}_{n} 0\right]^{\mathrm{T}}=\boldsymbol{\Phi}^{\mathrm{T}}(s) \mathbf{q}_{n}
\end{aligned}
$$

The matrix form of shape function $\Phi(s)$ both in PPIM and in RPIM are defined by

$$
\boldsymbol{\Phi}^{\mathrm{T}}(s)=\left[\phi_{1}(s), \phi_{2}(s), \cdots, \phi_{n}(s)\right]
$$

And equation (5) and (11) can be written as

$$
u(s)=\sum_{i=1}^{n} \phi_{i}(s) u_{i}, q(s)=\sum_{i=1}^{n} \phi_{i}(s) q_{i}
$$

The shape functions are formed by RPIM should be more 
The full set of equations for low-frequency electromagnetic field can be now written as

$$
\nabla \times \mathbf{E}=-\frac{\partial \mathbf{B}}{\partial t}, \nabla \times \mathbf{H}=\mathbf{J}_{e}+\mathbf{J}_{s}, \mathbf{B}=\mu \mathbf{H}, \mathbf{J}_{e}=\sigma \mathbf{E}
$$

where $\mathbf{J}_{e}$ and $\mathbf{J}_{s}$ are eddy current and source current respectively. The initial and boundary problems for two-dimensional transient eddy current field is written as

$$
\left\{\begin{aligned}
\nabla^{2} u & =\sigma \mu \frac{\partial u}{\partial t}-P & & \text { in } \Omega \text { at } 0<t<t_{n} \\
u & =\bar{u} & & \text { on } \Gamma_{u} \text { at } 0<t<t_{n} \\
q & =\bar{q} & & \text { on } \Gamma_{q} \text { at } 0<t<t_{n} \\
u & =u_{0} & & \text { in } \Omega \text { at } t=0
\end{aligned}\right.
$$




\section{IV .BPPIM and BRPIM Formulations}

A. Discrete equations by BPPIM and BRPIM

The well-known boundary integration equation for two-dimensional linear medium problems is given by

$$
\begin{array}{r}
c_{i}\left(u_{i}\right)_{t_{n}}+\frac{1}{\sigma \mu} \int_{0}^{t_{n}} \int_{\Gamma} q^{*} u \mathrm{~d} \Gamma \mathrm{d} t=\frac{1}{\sigma \mu} \int_{0}^{t_{n}} \int_{\Gamma} u^{*} q \mathrm{~d} \Gamma \mathrm{d} t \\
+\frac{1}{\sigma \mu} \int_{0}^{t_{n}} \int_{\Omega} u^{*} P \mathrm{~d} \Omega \mathrm{d} t+\left[\int_{\Omega} u^{*} u \mathrm{~d} \Omega\right]_{t=0} \\
u^{*}=\frac{\sigma \mu}{4 \pi\left(t_{n}-t\right)} \exp \left(-\frac{\sigma \mu r^{2}}{4 \pi\left(t_{n}-t\right)}\right) \\
q^{*}=-\frac{(\sigma \mu)^{2}}{8 \pi\left(t_{n}-t\right)^{2}} \exp \left(-\frac{\sigma \mu r^{2}}{4 \pi\left(t_{n}-t\right)}\right) \frac{\partial r}{\partial n}
\end{array}
$$


To get the numerical solution of equation (17), time should be discrete into $N$ time steps for computation

$$
u(t)=\sum_{k=1}^{l} M^{k} u^{k}=\mathbf{M}^{\mathrm{T}} \mathbf{u}^{t}, q(t)=\sum_{k=1}^{l} M^{k} q^{k}=\mathbf{M}^{\mathrm{T}} \mathbf{q}^{t}
$$

Where $u^{k}$ and $q^{k}$ are the values of $u$ and $q$ at time $t=t_{k}$, $\mathbf{M}^{\mathrm{T}}=\left[M^{1}, M^{2}, \cdots, M^{l}\right]$ are the shape functions of $u^{k}$ and $q^{k}$, $\mathbf{u}^{t}=\left[u^{1}, u^{2}, \cdots, u^{2}\right]$ and $\mathbf{q}^{t}=\left[q^{1}, q^{2}, \cdots, q^{2}\right]$.

Substituting (13) and (19) into (17) yields the boundary meshless method for all nodes on the boundary of the problem domain

$$
\mathbf{H}^{k} \mathbf{U}^{k}=\mathbf{G}^{k} \mathbf{Q}^{k}+\mathbf{F}{ }^{k} \mathbf{P}^{k}+\mathbf{B} \overline{\mathbf{U}}^{0}
$$

Where 


$$
\begin{array}{r}
\mathbf{H}^{k}=\mathbf{c}_{i}+\frac{1}{\sigma \mu} \int_{t_{k-1}}^{t_{k}} \int_{\Gamma} \mathbf{q}^{*} \boldsymbol{\Phi}^{\mathrm{T}}(s) \mathbf{M}^{\mathrm{T}} \mathrm{d} \Gamma \mathrm{d} t \\
\mathbf{G}^{k}=\frac{1}{\sigma \mu} \int_{t_{k-1}}^{t_{k}} \int_{\Gamma} \mathbf{u}^{*} \boldsymbol{\Phi}^{\mathrm{T}}(s) \mathbf{M}^{\mathrm{T}} \mathrm{d} \Gamma \mathrm{d} t \\
\mathbf{F}^{k}=\frac{1}{\sigma \mu} \int_{t_{k-1}}^{t_{k}} \int_{\Omega} \mathbf{u}^{*} \boldsymbol{\Phi}^{\mathrm{T}}(s) \mathbf{M}^{\mathrm{T}} \mathrm{d} \Omega \mathrm{d} t \quad \mathbf{B}=\left[\int_{\Omega} \mathbf{u}^{*} \mathrm{~d} \Omega\right]_{t=0} \\
\mathbf{U}^{k}=\left[u_{1}, u_{2}, \cdots, u_{n}\right]_{t=t_{k}}^{\mathrm{T}}, \mathbf{Q}^{k}=\left[q_{1}, q_{2}, \cdots, q_{n}\right]_{t=t_{k}}^{\mathrm{T}} \\
\mathbf{P}^{k}=\left[p_{1}, p_{2}, \cdots, p_{m}\right]_{t=t_{k}}^{\mathrm{T}}, \overline{\mathbf{U}}^{0}=\left[\bar{u}_{1}, \bar{u}_{2}, \cdots, \bar{u}_{m}\right]_{t=0}^{\mathrm{T}}
\end{array}
$$

B. Operation on singular integral of BPPIM and BRPIM

The log Gaussian quadrature are required to evaluate the $\ln (1 / x)$ type singular integrals, which are existed in matrix $\mathbf{G}$, as follows 


\section{are investigated to compare BPPIM and BRPIM.}

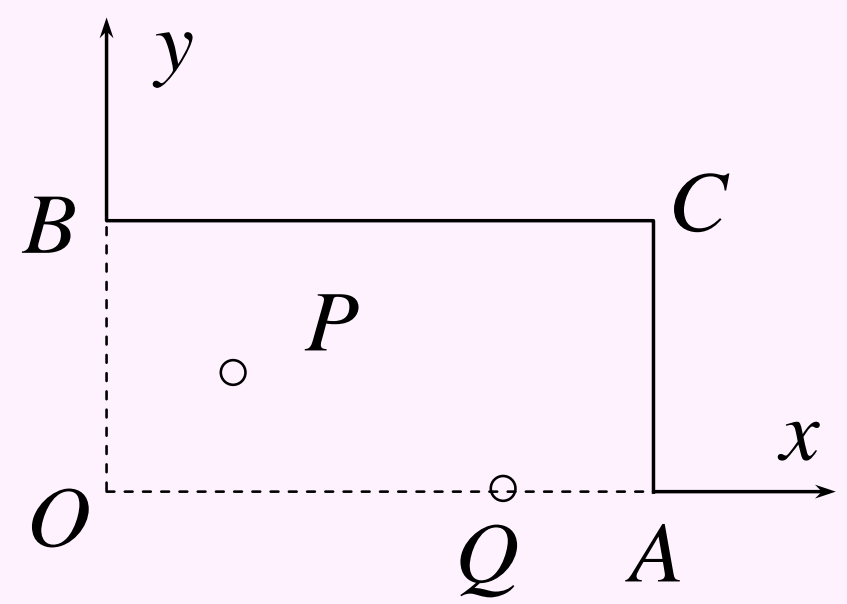

Fig.2 Cross section of one quadrant of the metal column

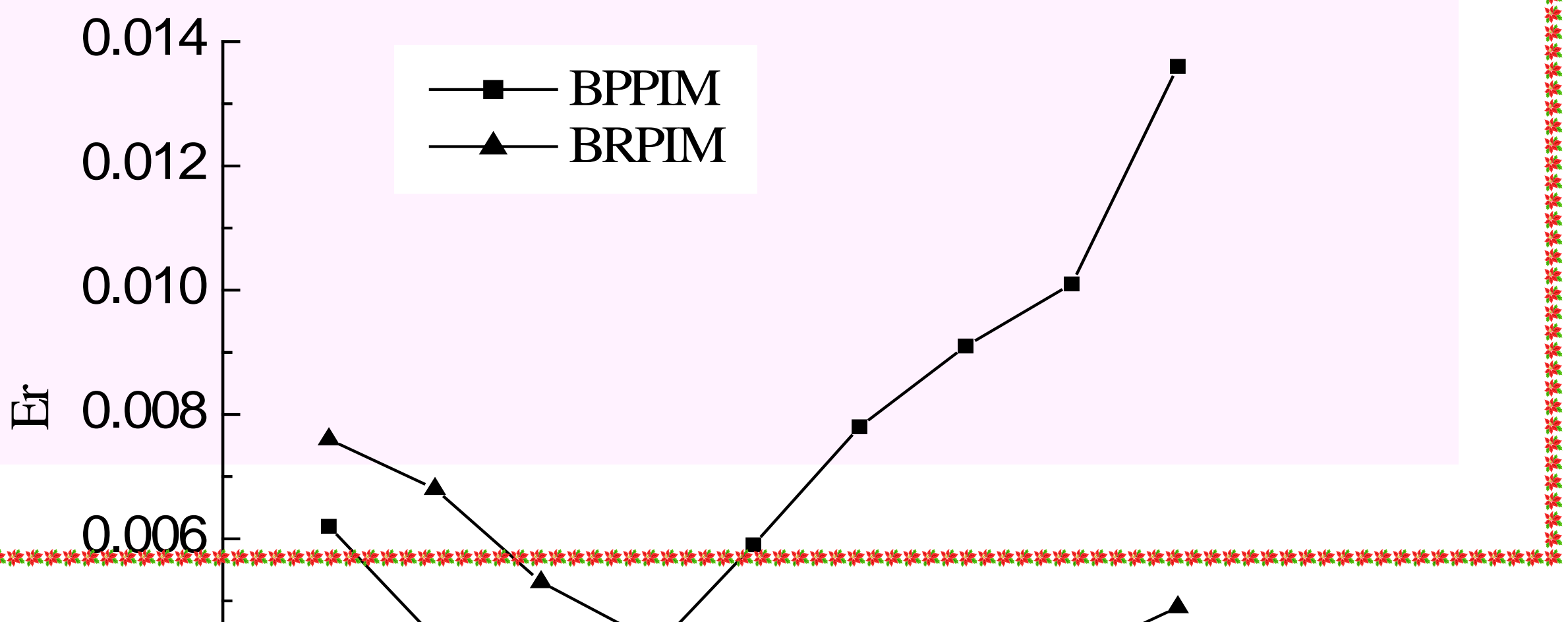




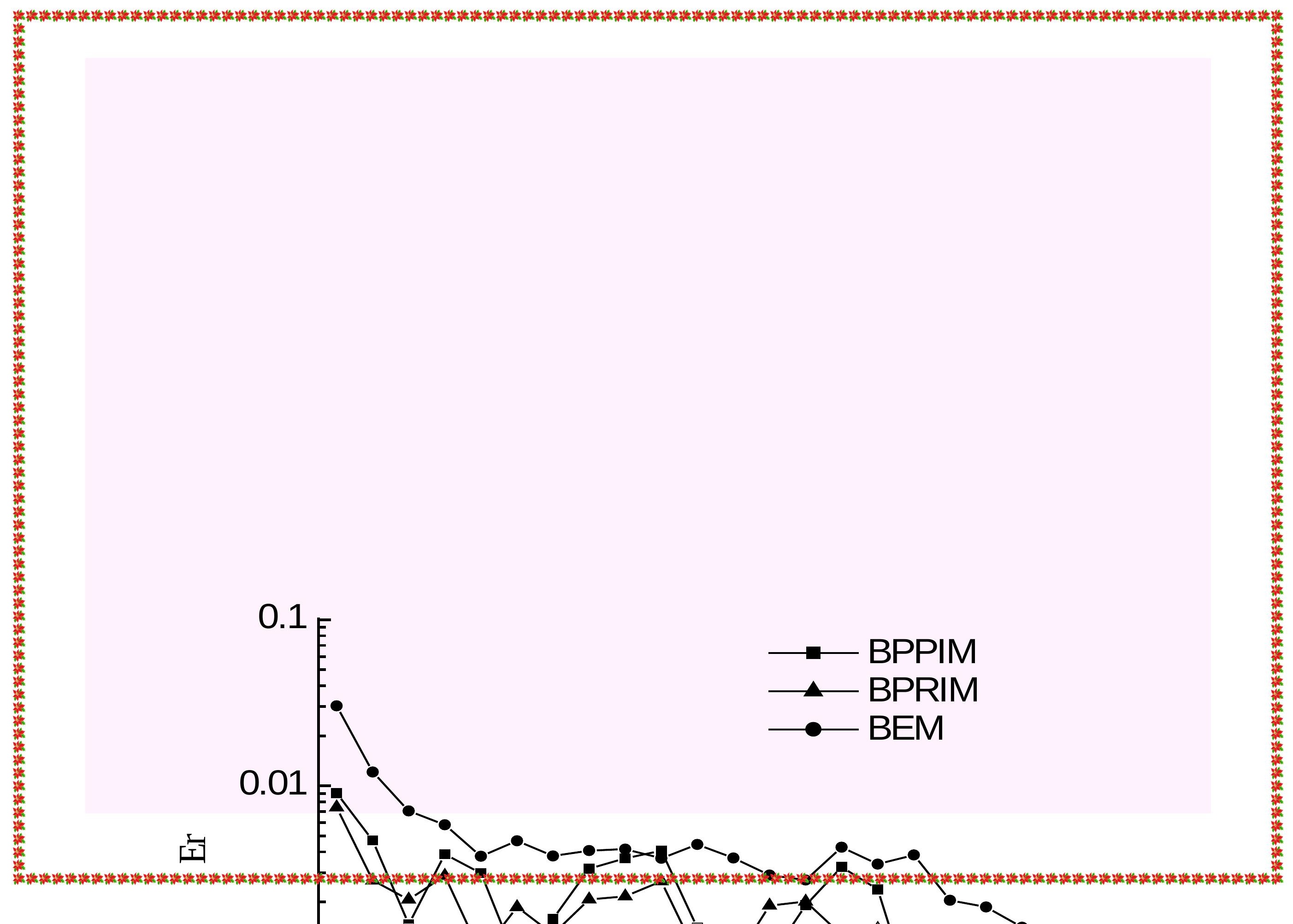




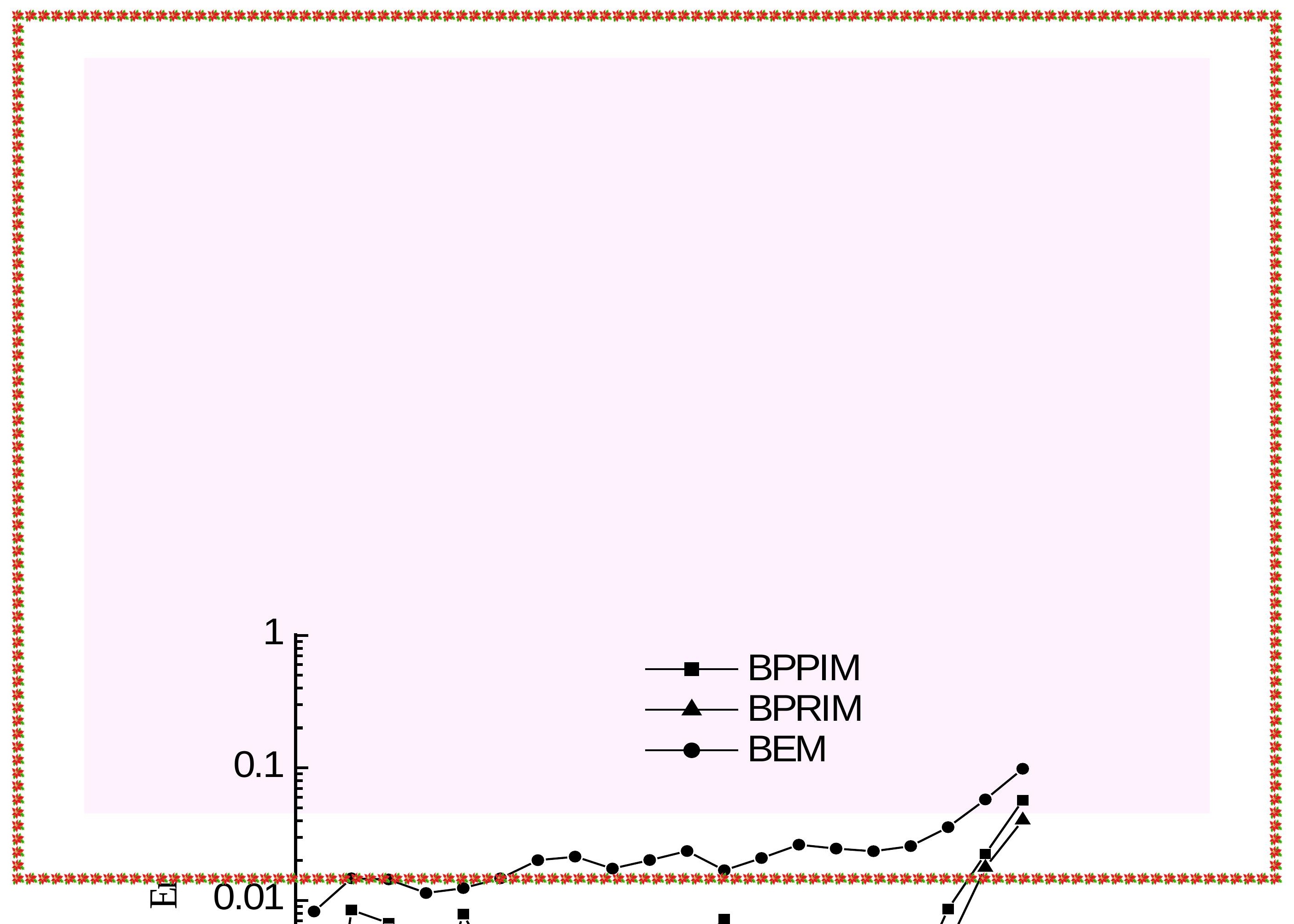




\section{CONCLUSIONS}

Both BPPIM and BRPIM belong to point interpolative boundary meshless method, they are both effective and suitable for 
